\title{
The Critical Role of the USP Performance Verification Test in Dissolution Testing and Qualification of the Paddle Apparatus
}

\author{
Will Brown*, Satish Perivilli, Doug Podolsky, Erika S. Stippler, and Steven Walfish \\ USP, Rockville, MD, USA
}

e-mail:WEB@usp.org

\begin{abstract}
Performance qualification of the United States Pharmacopeia (USP) paddle apparatus (USP apparatus 2), as described in USP General Chapter <711> Dissolution, requires a demonstration of the dissolution behavior of a standard material as well as control of the mechanically measurable parameters of the apparatus. The USP performance verification test (PVT), an integral part of $\langle 711\rangle$, plays an important role in demonstrating the apparatus suitability and addressing the interlaboratory variability of dissolution results by using a standard procedure and reference standard material. The USP has used a specially formulated tablet containing prednisone in the role of the reference standard material that has been shown to be sensitive without undue variability. This paper describes how the use of the PVT ensures the accuracy of dissolution results by understanding and controlling the variability. This paper provides an overview of the following: the dissolution test; the mechanics of the test and associated hydrodynamics; the PVT reference standard; PVT and mechanical qualification.
\end{abstract}

KEYWORDS: USP apparatus 2, paddle apparatus, dissolution, performance verification test, reference standard material, hydrodynamics

\section{INTRODUCTION}

his paper focuses on the value of the qualification of the paddle apparatus officially titled, "USP apparatus 2," but the overarching principles discussed here can be applied to other in vitro test apparatuses. The USP's current thinking is that qualification of a dissolution apparatus requires a demonstration of performance using the dissolution behavior of a standard material in addition to control of the mechanically measurable attributes. This position is driven by experience both with dissolution testing and the Performance Verification Test (PVT) and it is discussed in the following sections of this paper. Over the years, the USP has used a specially formulated tablet (containing prednisone) in the role of the reference standard (RS) material for the paddle apparatus. Data analysis from decades of collaborative studies with various iterations of RS tablets has enabled USP to observe dissolution results from laboratories worldwide. The wealth of data has led us to conclude that the elimination of variability arising from factors external to the dosage form in order to ensure validity of dissolution results remains an elusive goal $(1,2)$. This paper will demonstrate the important role that the PVT plays in ensuring the accuracy of dissolution results by identifying the sources of variability and subsequently, attempting to mitigate them.

A dissolution apparatus is expected to reproducibly promote drug release, dissolution, and mass transport from a dosage form. A dissolution assembly generally consists of a set of six individual vessel and stirring element combinations that are adjusted independently. Understanding the hydrodynamics in each vessel is critical to achieving reproducibility in results because changes in fluid flow may cause variability of results. There is a need for a sensitive and accessible probe to evaluate the effect of these changes in order to control and understand the variability in dissolution results due to apparatus performance. Differences in dissolution behavior of a standard tablet can indicate changes or differences in the fluid environment that the tablet is subjected to.

In addition, measurements by analytical instrumentation must be accurate to generate valid results. Evaluation of the accuracy of those measurements is an obligation of the analytical laboratory operating under current good 
manufacturing practices or International Organization for Standardization (ISO) requirements. According to ISO terminology, accuracy is comprised of trueness and precision and may be assessed by measurement of a standard material (3). The trueness is the result of the measurement compared to the value that is considered the true value; and precision is expressed in terms of the standard deviation. For the USP reference material, the accuracy of dissolution results is assessed using the acceptance ranges for geometric mean and coefficient of variation (CV) given in the certificate $(4,5)$.

The original use of USP RS tablets was necessitated by the variability observed in the dissolution results. From the beginning, the ranges of acceptable results established from collaborative studies have formed the basis for determining acceptable performance of dissolution apparatus and assemblies. Evaluations of the repeatability and reproducibility of data from participants in collaborative studies were used to estimate acceptable performance of the apparatus and assembly (i.e., dissolution results from USP Prednisone Tablets RS). The original approach for determining acceptable performance has largely been followed over time with refinements introduced to improve the test. The contribution of the variability inherent in the standard tablets to the variability of the PVT results is always a concern; however, statistical analysis of the collaborative study results allows the evaluation of the variability of the RS to be separated from the overall variability (6). The current USP reference material was shown to perform consistently and with acceptable variation.

One of the challenges with the use of any material in the PVT is the balance of sensitivity and variability. These two important attributes of a PVT material are characterized by variability of dissolution results. Sensitivity to variations in conditions of the apparatus/assembly is a desired property of the material and it is observed through variability of results. Other variability (such as from medium preparation or from the samples under investigation) confounds sensitivity and is a concern associated with a PVT. The diagnostic value in the PVT result using USP RS tablets is that the sampling time will fall in a sensitive portion of the dissolution profile, between $30 \%$ and $70 \%$ dissolution, which is typically associated with high variability. The USP product has been shown to have considerably lower variability than acceptable marketed immediate-release products in that portion of their dissolution range(s) (7).
Enhancement of dimensional and operational tolerances for dissolution apparatus has been advanced as an adequate means of controlling variability of dissolution results. However, without a demonstration that the apparatus can produce acceptable results in action, the application of such enhanced mechanical control falls short of performance qualification (PQ), which is part of analytical apparatus qualification.

\section{DISCUSSION}

There are many considerations in choosing a suitable probe to evaluate the source of variability in dissolution results. This begins with an understanding of the dissolution test that includes, but is not limited to, the following: the apparatus setup and its operation; other factors that influence dissolution; the governing hydrodynamics; and inherent variability of the sample. The following subsections discuss each of these topics in detail.

\section{Dissolution Test Overview}

A dissolution experiment shows the release of a drug substance from a dosage form to solution as a function of time. The change of the drug substance's concentration in the solution is taken as the indication of drug release from the dosage form and dissolution. At its simplest, the dissolution test selects against drug products that are unlikely to perform adequately in vivo.

The in vitro dissolution methodology is used over the entire lifecycle of a drug product from the development phase for optimization of the drug product performance to the evaluation of post-approval changes and stability as well as confirmation of the marketed-product performance. Following approval, the dissolution test provides the primary evidence of continuously acceptable performance of successive lots of the marketed product. In that role, the dissolution test targets the true variability that characterizes product performance. Attempts to link the dissolution profile to pharmacokinetic parameters are expected by regulatory agencies. Through dissolution profile comparison, dissolution performance can be used to indicate bioequivalence $(8-10)$.

A simplified dissolution test becomes part of the commitment within a marketing authorization. This simplified test is used in quality control and as a determinant of shelf life. For immediate-release products, the criteria are in a form that evaluates failure to achieve a certain percentage of the label claim ( $Q$ value) dissolved at the specified time. As systematic perturbations in the paddle apparatus performance tend to accelerate 
dissolution, establishment that the system is under control is essential $(11,12)$. Drug product manufacturers as well as regulatory agencies depend on valid dissolution results to demonstrate and ensure efficacy and safety of drug products.

\section{Mechanics of the Test and Associated Hydrodynamics}

As a preface to describing hydrodynamics in USP apparatus 2, an understanding of the apparatus setup and operation is necessary. The apparatus consists of a paddle-shaft combination (i.e., impeller) rotating inside a cylindrical vessel with a hemispherical bottom at a specified stirring rate measured in revolutions per minute (rpm) for a specified amount of time. An assembly consists of a set of impeller/vessel combinations with controls for the fluid temperature in the vessels. At the beginning of a dissolution run, a dosage form is usually positioned at the bottom of each vessel. The fluid motion caused by the rotation of the impeller facilitates dissolution of the drug substance from the formulation. For the following discussion, it is assumed that all impellers within an assembly rotate at the same rpm and that the media temperature in each vessel is constant across the assembly.

The dissolution behavior of the drug substance is influenced by 1) fluid flow behavior in the vessel (hydrodynamics); 2) physical and chemical characteristics of the dosage form; 3 ) dissolution media properties; and 4) media preparation procedure such as deaeration. Considering that the latter three are retained across a dissolution test, understanding the hydrodynamics becomes critical. Hydrodynamics is affected by the design, setup, and operational parameters of the apparatus along with the location of the dosage form in the vessel.

In addition to the vessel and impeller dimensions, the key geometric factors that influence the apparatus' hydrodynamics are the centering, wobble, and verticality of the impeller along with the distance from the impeller to the vessel bottom. Any differences in vessel-to-vessel hydrodynamics within the same assembly would arise from differences in the relative positions of the vessel to the impeller, differences in vessel surface irregularity, and position-specific disturbances. These disturbances can also include vibration from heating device, water bath, and other external sources. It is critical to describe and understand the combined influence of all the parameters mentioned above on the hydrodynamics because the hydrodynamics in a vessel is the environment that a dosage form is exposed to and that ultimately defines the dissolution behavior. Keeping the environment similar across vessels within an assembly will ensure that the dosage form in each vessel will respond similarly to one another. Additionally, this expectation needs to be extended for different assemblies around the globe and from test to test to ensure validity of the dissolution results.

The following describes the characteristic hydrodynamics within a vessel-impeller combination. In the paddle apparatus, the dosage form is typically located at the bottom of the vessel. If the vessel is perfectly cylindrical with a perfectly hemispherical bottom, the dosage form will move toward the center of the vessel bottom due to gravity. The hydrodynamics in the vessel is characterized by tangentially driven flows owing to the rotation of the impeller; the tangential components of velocity dominate the axial and radial components. Additionally, the hydrodynamics in apparatus 2 is characterized by two recirculation loops on either side of the impeller's vertical axis - one above and another below the paddle - and by a low velocity "dead zone" located around the dosage form's location, directly beneath the center of the paddle. Velocity magnitude vectors on a central vertical plane obtained from computational fluid dynamics (CFD) simulations, which used a steady-state pseudo-transient model for paddle apparatus at $50 \mathrm{rpm}$, with $900 \mathrm{~mL}$ of water at $37^{\circ} \mathrm{C}$ in a vessel, are shown in Figure 1. It is expected that deviations from the ideal setup (i.e., ideal geometry and operation with no external disturbances) will change fluid flow behavior in the vessel. Studies on hydrodynamics, as affected by changes of individual parameters, have been published in literature. These include rotational speed, impeller offset and distance of paddle to vessel bottom $(13,14)$. Some of these parameters have been demonstrated to influence hydrodynamics more than others as an individual factor. The authors of this paper are not aware of any studies that have discussed combination effects of these parameters and/or external factors such as vibration. Even with information available from studies on individual parameters - and realizing that the combination effects are going to be more complex - it is easy to recognize the need for a probe to measure the changes in hydrodynamics. In summary, a probe that captures differences in the fluid flow behavior arising from differences in the geometric/operational settings or external factors is useful. 


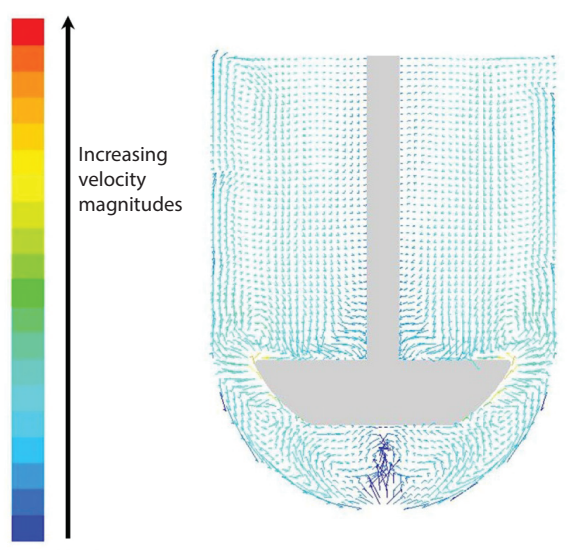

Figure 1. Representative velocity magnitude vectors with fixed number of samples (in-plane vectors shown with fixed length).

\section{Variability of PVT Reference Standard}

USP Prednisone Tablets RS were introduced in 1978 to address the interlaboratory variability of dissolution results (15-17). The different sources of variability in the PVT testing include, but are not limited to, run-to-run, within-run, and tablet-to-tablet variability. The variability between laboratories and instruments indicates that any product testing on multiple instruments in multiple locations will have higher variability than the variability seen within the laboratory on a single instrument. These sources of variability are not attributable to the sample under investigation. This is true not only for the PVT but for any dissolution test for any sample.

The acceptance criteria for each lot of USP Prednisone Tablets RS have depended on a collaborative study as the source of data. The statistical methods have been refined over the years. Initially, analysis of variance was used to determine the mean within-laboratory variability, with the grand mean of the submitted data calculated as the arithmetic mean of the data. A combined standard deviation and a coverage factor to develop a prediction interval for individual results of future testing were generated from the variance components (18). This approach was followed with some modification in the subsequent studies for replacement lots of USP Prednisone Tablets RS (19-21). A refinement in this approach using restricted maximum likelihood estimates was reported for the USP Lot POE203 USP Prednisone Tablets RS collaborative study (22). In 2009, the acceptreject approach for dissolution was changed from a perposition result to the basis of the mean and variability of a result for a dissolution assembly using the USP Prednisone Tablets RS $(4-6,23,24)$.

In June 2005, USP had 28 laboratories participate in the USP Lot P0E203 USP Prednisone Tablets RS collaborative study (22). This study showed that the average within-run CV was 8.5 for the paddle apparatus. A collaborative study, conducted in 2017 for Lot R072M0 with 18 laboratories, determined that the average within-run CV values for the USP Prednisone Tablets RS was 5.0 (unpublished data, 2017). Additional data from internal studies show consistent performance. The internal data were used to calculate the typical variability for a PVT run. Multiple runs per day, performed over several days for a six-vessel instrument, showed an average within-vessel CV of 3.9\% for the paddle apparatus. This estimate accounts for the day-to-day and vessel-to-vessel variability and is also a surrogate for tablet-to-tablet variability. Additionally, the same data showed the performance run-to-run and within-run values indicated in Table 1.

Table 1. Typical CV values for a PVT run of USP Prednisone Tablets $R S$, calculated from internal studies.

\begin{tabular}{|c|c|c|}
\hline Within-Run CV & Run-to-Run CV & Total CV \\
\hline $4.0 \%$ & $2.2 \%$ & $5.6 \%$ \\
\hline
\end{tabular}

$C V$, coefficient of variation; $P V T$, performance verification test; USP, United States Pharmacopeia.

Published data show that the variability of the USP Prednisone Tablet RS is less than commercially available tablet products when compared in the sensitive early part of the dissolution profile (7). Variability of results for USP Prednisone Tablets RS from internal and external collaborative studies shows consistent performance within the lot. Studies in which operational or mechanical parameters of the dissolution equipment were varied show an increase in the within-run variability, indicating that USP Prednisone Tablets RS can be used to detect changes in operation of dissolution assemblies (11).

\section{PVT and Mechanical Qualification}

USP apparatus 2 is similar in form to any simple blending equipment that has a source of agitation and a vessel to contain the material being blended. Certainly, the uniform distribution of dissolved material within the volume being stirred is one function of this device. In addition to this function, the apparatus is intended to provide a repeatable and reproducible environment for the dissolution process at the interface of the dissolution medium and the dosage form. In order for these two functions to occur repeatedly within one vessel as well as among the multiple vessels in a six or more position test assembly, the dimensional and other operational parameters must be held to an adequate level of standardization. The apparatus description and operational parameters in the USP General Chapter $<711>$ Dissolution provide the current official guidance 
for these considerations (25). Various documents have been published recommending narrower parameter ranges than those given in $<711>(26-28)$. Even though the narrower ranges ensure a tighter control of these parameters, which could aid in minimizing their effects on variability in dissolution results, they will not, by themselves, meet the expectation for overall evaluation of the dissolution apparatus' performance (they do not include the effect of external factors). The ranges currently stated in $<711>$ represent values harmonized with other major pharmacopeias (25). Though, as datadriven suggestions for improvement of the content of $<711>$ are received, the content of the chapter can be considered for revision.

\section{Design Functions of the Paddle Apparatus}

UThe two design functions of the paddle apparatus (i.e., controlled dissolution conditions and bulk mixing) can best be demonstrated in action. Analytical instrument qualification (AIQ) uses the progression from design qualification, installation qualification, and operational qualification (OQ), culminating in $P Q$ to assure "fitness for purpose" (29). OQ involves the evaluation and adjustment of mechanical and operational parameters one setting at a time. Each of these settings is expected to be within a certain tolerance, either provided in $\langle 711\rangle$ or by an alternative specification. In practice, the tolerances for each setting represent a safety zone whether confirmed by experience or simply assumed. Such a point estimate of the physical condition of the apparatus by $\mathrm{OQ}$ procedures falls short of the full application AIQ if the demonstration of repeatable and reproducible performance (i.e., $P Q$ ) is ignored. This is especially critical with a multiple test position dissolution assembly (11).

Current approaches that rely solely on mechanical qualification assume that the vessel qualities as manufactured are satisfactory and negligible as sources of variability. This assumption does not account for variability in inner surface uniformity and conformance to the ideal Euclidean surfaces (i.e., cylinder and hemisphere) (30-32). These irregularities potentially impact the hydrodynamics in the vessel that lead to varied results, as noted earlier in the section, Mechanics of the Test and Associated Hydrodynamics. OQ also does not evaluate or control external forces and disturbances that may affect dissolution results such as environmental vibration $(1,12,33-37)$. Currently, <711> does not define an approach for monitoring vibration and therefore does not define limits for vibration variables (25). Studies are ongoing to define limit recommendations.
We have concern regarding formal adoption of enhanced dimensional parameter tolerances where such enhancements are not solidly backed by data that reflect a public health necessity for the change. Without a link to dissolution performance, making enhanced parameters official may have the unintended consequence of forcing the removal of otherwise suitable dissolution apparatus from use. As the practice of dissolution is worldwide, the effect of such changes may fall disproportionately on laboratories that have limited resources available for apparatus replacement; however, if a dissolution apparatus can produce acceptable results in the PVT, it is suitable for dissolution testing.

Given their differences in formulation, manufacture, and size, all dosage forms will respond to the environment provided by the apparatus for testing. For example, the likelihood is higher that a formulation will dissolve faster if located outside of the dead zone than within the dead zone (38). Currently, USP Prednisone Tablets RS tablets are widely used as a probe to evaluate repeatability and reproducibility of the dissolution conditions provided by the paddle apparatus.

PVT ensures that the instrument is operating inside certain ranges even when the mechanical qualification is not conducted. Conversely, failing PVT indicates the presence of one or a combination of uncontrolled factors, such as improper environment, operation, setup, and analyst performance, even when mechanical qualification has been conducted. Considering the complex nature of possible interaction(s) among these factors, the challenge is to identify the specific cause(s) of the failure.

\section{CONCLUSION}

The dissolution test is an important measure of in vitro drug product performance and is relied upon by manufacturers and regulators to produce valid results. The dissolution or transfer of the drug substance into an appropriate liquid medium provides the measure of in vitro performance.

There are many factors that affect the dissolution behavior of a formulation such as the test setup and operational conditions. These factors affect the hydrodynamics that a formulation is subjected to, which in turn might present as differences in dissolution behavior. A probe that recognizes deviations from a standard "specified" setup is useful as a means of ensuring valid dissolution test results. The USP has employed dissolution results from a tablet formulation in this role for 4 decades. Results for a USP PVT will be subjected to sources of variability that 
will include the contribution of the dissolution apparatus and assembly. This is true for the dissolution of any product whether manufactured as a medicine or as a probe of dissolution test setup. The variability of results is representative of variability for any dissolution sample.

The USP conducts a multiple center collaborative study to provide the data for the estimation of the true ranges for each new lot of its RS tablets. The observed variability not attributable to the sample is limited by the acceptance ranges given with the USP reference material. Sensitivity and variability are necessarily intertwined in a performance verification reference material. The USP Prednisone Tablets RS have been shown to be sensitive without undue variability.

PQ using a probe sensitive to changes in apparatus operation is a necessary part of AIQ for dissolution apparatus. Although dimensional and operational fidelity to documented requirements is an important consideration, a demonstration that the apparatus and assembly can produce acceptable results during $P Q$ is necessary to remove doubt regarding its suitability for use. Accordingly, the USP remains open to data-driven requests for revision of the dimensional and operational requirements in $<711>$.

\section{CONFLICT OF INTEREST}

The authors are USP employees. USP has financial interests in the PVT program and the distribution of Prednisone Tablets RS.

\section{REFERENCES}

1. Crist, B.; Spisak, D. Evaluation of induced variance of physical parameters on the calibrated USP dissolution apparatus 1 and 2. Dissolut. Technol. 2005, 12, 28-31. DOI: 10.14227/ DT120105P28.

2. Mirza, T. Mechanical versus chemical dissolution calibration. Dissolut. Technol. 2000, 7, 6-7. DOI: 10.14227/DT070100P6.

3. ISO 5725-6:1994 Accuracy (trueness and precision) of measurement methods and results-Part 6: Use in practice of accuracy values. International Organization for Standardization (ISO). Published December 1994. Accessed November 28, 2018. https://www.iso.org/standard/11837.html.

4. DeStefano, A. J.; Hauck, W. W.; Stippler, E. S.; Brown, W. E.; Li, C.; Huang, C. G.; Jones, B. J.; O'Hool, K.; Koch, W. F.; Williams, R. L. Establishing new acceptance limits for dissolution performance verification of USPC apparatus 1 and 2 using USPC prednisone tablets reference standard. Pharm. Res. 2011, 28, 505-516. DOI: 10.1007/s11095-010-0295-3.

5. Hauck, W. W.; DeStefano, A. J.; Brown, W. E.; Stippler, E. S.; Abernethy, D. R.; Koch, W. F.; Williams, R. L. Change in criteria for USP dissolution performance verification tests. AAPS PharmSciTech. 2009, 10, 21-26. DOI: 10.1208/s12249-0089169-y.

6. Hauck, W. W.; Li, C.; Stippler, E. S.; Brown, W. E. Establishing acceptance limits for dissolution performance verification of USP apparatus 1 and 2 using USP prednisone tablets reference standard lot Q0H398. Dissolut. Technol. 2013, 20, 6-10. DOI: 10.14227/DT200113P6.

7. Nithyanandan, P.; Hauck, W. W.; Munoz, J.; Deng, G.; Brown, W.; Manning, R. G.; Wahab, S. Dissolution variability: comparison of commercial dosage forms with US Pharmacopeia lot $P$ prednisone reference standard tablets-a technical note. AAPS PharmSciTech. 2008, 9, 238-242. DOI: 10.1208/s12249-0089034-z.

8. Dissolution Testing of Immediate Release Solid Oral Dosage Forms; Guidance for Industry. U.S. Department of Health and Human Services, Food and Drug Administration, Center for Drug Evaluation and Research (CDER), U.S. Government Printing Office: Washington, DC, 1997.

9. Immediate Release Solid Oral Dosage Forms Scale-Up and Postapproval Changes: Chemistry, Manufacturing, and Controls, In Vitro Dissolution Testing, and In Vivo Bioequivalence Documentation; Guidance for Industry. U.S. Department of Health and Human Services, Food and Drug Administration, Center for Drug Evaluation and Research (CDER), U.S. Government Printing Office: Washington, DC, 1995.

10. Waiver of In Vivo Bioavailability and Bioequivalence Studies for Immediate-Release Solid Oral Dosage Forms Based on a Biopharmaceutics Classification System; Guidance for Industry. U.S. Department of Health and Human Services, Food and Drug Administration, Center for Drug Evaluation and Research (CDER), U.S. Government Printing Office: Washington, DC, 2017.

11. Eaton, J.; Deng, G.; Hauck, W. W.; Brown, W.; Manning, R. G.; Wahab, S. Perturbation study of dissolution apparatus variables-a design of experiment approach. Dissolut. Technol. 2007, 14, 20-26. DOI: 10.14227/DT140107P20.

12. Subcommittee on Dissolution Calibration, Pharmaceutical Research and Manufacturers of America (PhRMA) Dissolution Calibration: Recommendations for reduced chemical testing and enhanced mechanical calibration. Pharm. Forum. 2000, 26, 1149-1166.

13. Bai, G.; Wang, Y.; Armenante, P. M. Velocity profiles and shear strain rate variability in the USP dissolution testing apparatus 2 at different impeller agitation speeds. Int. J. Pharm. 2011, 403, 1-14. DOI: 10.1016/j.ijpharm.2010.09.022.

14. Bai, G.; Armenante, P. M. Velocity distribution and shear rate variability resulting from changes in the impeller location in the USP dissolution testing apparatus II. Pharm. Res. 2008, 25, 320336. DOI: 10.1007/s11095-007-9477-z.

15. Brown, C. K.; Buhse, L.; Friedel, H. D.; Keitel, S.; Kraemer, J.; Morris, J. M.; Stickelmeyer, M.; Yomota, C.; Shah. V. P. FIP position paper on qualification of paddle and basket dissolution apparatus. 
AAPS PharmSciTech. 2009, 10, 924-927. DOI: 10.1208/s12249009-9291-5.

16. Cabana, B. E.; O'Neill, R. FDA's report on drug dissolution. Pharm. Forum. 1980, 6, 71-89.

17. Section for Official Laboratories and Medicines Control Services and the Section of Industrial Pharmacists of the International Pharmaceutical Federation (FIP). FIP guidelines for dissolution testing of solid oral products final draft. Pharm. Forum. 1995, 21, 1371-1382.

18. Sarapu, A. C.; Lewis, A. R.; Grostic, M. F. Analysis of PMA collaborative studies of dissolution test calibrators. Pharm. Forum. 1980, 6, 172-176.

19. PhRMA Quality Assurance Section Dissolution Committee. The USP dissolution calibrator tablet collaborative study-an overview of the 1994 process. Pharm. Forum. 1994, 20, 81778201.

20. PhRMA Quality Assurance Section Dissolution Committee. The USP dissolution calibrator tablet collaborative study-an overview of the fourth quarter 1994 process. Pharm. Forum. 1995, 21, 841-858.

21. Pharmaceutical Research and Manufacturers of America. The USP dissolution calibrator tablet collaborative study-an overview of the 1996 process. Pharm. Forum. 1997, 23, 41984237.

22. Glasgow, M.; Dressman, S.; Brown, W.; Foster, T.; Schuber, S.; Manning, R. G.; Wahab, S. Z.; Williams, R. L.; Hauck, W. W. The USP performance verification test, part II: collaborative study of USP's lot P prednisone tablets. Pharm. Res. 2008, 25, 11101115. DOI: 10.1007/s11095-007-9482-2.

23. Hauck W. W.; Cecil T. L.; Brown, W.; Abernethy, D. R.; Koch, W.F.; Williams, R. L. USP responses to comments on stimuli article, "Proposed change to acceptance criteria for dissolution performance verification testing." Pharm. Forum. 2008, 34, 474-476.

24. Hauck, W. W.; Manning, R. G.; Cecil, T. L.; Brown, W.; Williams, R. L. Proposed change to acceptance criteria for dissolution performance verification testing. Pharm. Forum. 2007, 33, 574579 .

25. <711> Dissolution. In The United States Pharmacopeia and National Formulary USP 41-NF 36. The United States Pharmacopeial Convention, Inc.: Rockville, MD, 2018; pp 64596469.

26. ASTM E2503-13e1, Standard Practice for Qualification of Basket and Paddle Dissolution Apparatus. ASTM International: West Conshohocken, PA, 2013. DOI: 10.1520/E2503-13E01.
27. Dissolution Toolkit Procedures for Mechanical Calibration and Performance Verification Test Apparatus 1 and Apparatus 2. Version 2.0. The United States Pharmacopeial Convention, Inc.: Rockville, MD, 2010. Accessed November 28, 2018. http://www. usp.org/sites/default/files/usp/document/our-work/referencestandards/dissolution-toolkit-version2-portuguese.pdf.

28. The Use of Mechanical Calibration of Dissolution Apparatus 1 and 2-Current Good Manufacturing Practice (CGMP); Guidance for Industry. U.S. Department of Health and Human Services, Food and Drug Administration, Center for Drug Evaluation and Research (CDER), U.S. Government Printing Office: Washington, DC, 2010.

29. < 1058> Analytical Instrument Qualification. The United States Pharmacopeia and National Formulary USP 41-NF 36. The United States Pharmacopeial Convention, Inc.: Rockville, MD, 2018; pp 7005-7011.

30. Liddell, M.R.; Deng, G.; Hauck, W.W. Dissolution testing variability: effect of using vessels from different commercial sources. Am. Pharm. Rev. 2007, 10, 122-128.

31. Liddell, M. R.; Deng, G.; Hauck, W. W.; Brown, W. E.; Wahab, S. Z.; Manning, R. G. Evaluation of glass dissolution vessel dimensions and irregularities. Dissolut. Technol. 2007, 14, 28-33. DOI: 10.14227/DT140107P28.

32. Tanaka, M.; Fujiwara, H: Fujiwara, M. Effect of the irregular inner shape of a glass vessel on prednisone dissolution results. Dissolut. Technol. 2005, 12, 15-19. DOI: 10.14227/DT120405P15.

33. Oates, M. Recent innovations in dissolution calibration. Dissolut. Technol. 1999, 6, 11. DOI: 10.14227/DT060399P11.

34. Collins, C. C. VIBRATION: what is it and how might it affect dissolution testing? Dissolut. Technol. 1998, 5, 16-18. DOI: 10.14227/DT050498P16.

35. Gao, Z.; Thies, A.; Doub, W. Vibration effects of lab equipment on dissolution testing with USP paddle method. J. Pharm. Sci. 2010, 99, 403-412. DOI: 10.1002/jps.21847.

36. Gao, Z.; Moore, T. W.; Buhse, L. F.; Doub, W. H. The random vibration effects on dissolution testing with USP apparatus 2. J. Pharm. Sci. 2008, 98, 297-306. DOI: 10.1002/jps.21402.

37. Gao, Z.; Moore, T. W.; Doub, W. H. Vibration effects on dissolution tests with USP apparatuses 1 and 2. J. Pharm. Sci. 2008, 97, 3335-3343. DOI: 10.1002/jps.21242.

38. Healy, A. M.; McCarthy, L. G.; Gallagher, K. M.; Corrigan, O. I. Sensitivity of dissolution rate to location in the paddle dissolution apparatus. J. Pharm. Pharmacol. 2002, 54, 441-444. DOI: 10.1211/0022357021778529. 